Article

\title{
Fractional integrals inequalities for exponentially $m$-convex functions
}

\author{
Sajid Mehmood ${ }^{1, *}$ and Ghulam Farid ${ }^{1}$ \\ 1 Department of Mathematics, COMSATS University Islamabad, Attock Campus, Pakistan.; \\ ghlmfarid@cuiatk.edu.pk(G.F) \\ * Correspondence: smjg227@gmail.com
}

Received: 18 October 2019; Accepted: 9 February 2020; Published: 24 March 2020.

\begin{abstract}
Fractional integral operators are very useful in mathematical analysis. This article investigates bounds of generalized fractional integral operators by exponentially $m$-convex functions. Furthermore, a Hadamard type inequality have been analyzed and, special cases of established results have been discussed.
\end{abstract}

Keywords: Convex function, exponentially $m$-convex functions, fractional integrals operators, bounds.

MSC: 26A51, 26A33, $26 \mathrm{D} 15$.

\section{Introduction}

$\mathbf{F}$ ractional integral operators play a vital role in the advancement of mathematical inequalities and many integral inequalities have been established in literature. In [1], Farid established the bounds of the Riemann-Liouville fractional integral operators for convex function. For more information related to fractional integral inequalities, the readers are referred to [2-10].

Definition 1. Let $\psi \in L_{1}[a, b]$ with $0 \leq a<b$. Then the left-sided and right-sided Riemann-Liouville fractional integral operators of a function $\psi$ of order $\sigma>0$ are defined as follows:

$$
\zeta_{a^{+}}^{\sigma} \psi(u)=\frac{1}{\Gamma(\sigma)} \int_{a}^{u}(u-\xi)^{\sigma-1} \psi(\xi) d \xi, \quad u>a
$$

and

$$
\zeta_{b^{-}}^{\sigma} \psi(u)=\frac{1}{\Gamma(\sigma)} \int_{u}^{b}(\xi-u)^{\sigma-1} \psi(\xi) d \xi, \quad u<b,
$$

where $\Gamma(\sigma)$ is the Gamma function defined as $\Gamma(\sigma)=\int_{0}^{\infty} t^{\sigma-1} e^{-t} d t$.

In [9], Mubeen et al. defined the following Riemann-Liouville $k$-fractional integral operators;

Definition 2. Let $\psi \in L_{1}[a, b]$ with $0 \leq a<b$. Then the left-sided and right-sided Riemann-Liouville $k$-fractional integral operators of a function $\psi$ of order $\sigma, k>0$ are defined as follows;

$$
\zeta_{a^{+}}^{\sigma, k} \psi(u)=\frac{1}{k \Gamma_{k}(\sigma)} \int_{a}^{u}(u-\xi)^{\frac{\sigma}{k}-1} \psi(\xi) d \xi, \quad u>a
$$

and

$$
\zeta_{b^{-}}^{\sigma, k} \psi(u)=\frac{1}{k \Gamma_{k}(\sigma)} \int_{u}^{b}(\xi-u)^{\frac{\sigma}{k}-1} \psi(\xi) d \xi, \quad u<b,
$$

where $\Gamma_{k}(\sigma)$ is the $k$-Gamma function defined as $\Gamma_{k}(\sigma)=\int_{0}^{\infty} t^{\sigma-1} e^{-\frac{t^{k}}{k}} d t$.

In [11], generalized Riemann-Liouville fractional integral operators are given as follows;

Definition 3. Let $\psi \in L_{1}[a, b]$ with $0 \leq a<b$ and $\phi$ be an increasing and positive function on $(a, b]$ having a continuous derivative $\phi^{\prime}$ on $(a, b)$. Then the left-sided and right-sided generalized Riemann-Liouville 
fractional integral operators of a function $\psi$ with respect to another function $\phi$ on $[a, b]$ of order $\sigma>0$ are defined as follows;

$$
\zeta_{\phi, a^{+}}^{\sigma} \psi(u)=\frac{1}{\Gamma(\sigma)} \int_{a}^{u}(\phi(u)-\phi(\xi))^{\sigma-1} \phi^{\prime}(\xi) \psi(\xi) d \xi, u>a
$$

and

$$
\zeta_{\phi, b^{-}}^{\sigma} \psi(u)=\frac{1}{\Gamma(\sigma)} \int_{u}^{b}(\phi(\xi)-\phi(u))^{\sigma-1} \phi^{\prime}(\xi) \psi(\xi) d \xi, u<b .
$$

In [7], Kwun et al. defined the generalized Riemann-Liouville $k$-fractional integral operators as follows;

Definition 4. Let $\psi \in L_{1}[a, b]$ with $0 \leq a<b$ and $\phi$ be an increasing and positive function on $(a, b]$ having a continuous derivative $\phi^{\prime}$ on $(a, b)$. Then the left-sided and right-sided generalized Riemann-Liouville $k$-fractional integral operators of a function $\psi$ with respect to another function $\phi$ on $[a, b]$ of order $\sigma, k>0$ are defined as follows:

$$
\zeta_{\phi, a^{+}}^{\sigma, k} \psi(u)=\frac{1}{k \Gamma_{k}(\sigma)} \int_{a}^{u}(\phi(u)-\phi(\xi))^{\frac{\sigma}{k}-1} \phi^{\prime}(\xi) \psi(\xi) d \xi, u>a
$$

and

$$
\zeta_{\phi, b^{-}}^{\sigma, k} \psi(u)=\frac{1}{k \Gamma_{k}(\sigma)} \int_{u}^{b}(\phi(\xi)-\phi(u))^{\frac{\sigma}{k}-1} \phi^{\prime}(\xi) \psi(\xi) d \xi, u<b .
$$

For suitable settings of $\phi$ and $k$, some interesting consequences can be achieved which are given in following remark;

Remark 1. 1. For $k=1$, (7) and (8) fractional integrals coincide with (5) and (6) fractional integrals.

2. By taking $\phi$ as identity function, (7) and (8) fractional integrals coincide with (1) and (2) fractional integrals.

3. For $k=1$, along with $\phi$ as identity function, (7) and (8) fractional integrals coincide with (3) and (4) fractional integrals.

4. For $\phi(u)=\frac{u^{1+s}}{1+s},(7)$ and (8) produce conformable fractional integrals given in [12].

5. For $k=1$, along with $\phi(u)=\frac{u^{\rho}}{\rho}, \rho>0$, (7) and (8) produce Katugampola fractional integrals given in [2].

6. For $k=1$, along with $\phi(u)=\frac{u^{\tau+s}}{\tau+s}$, (7) and (8) produce conformable fractional integrals given in [13].

7. For $\phi(u)=\frac{(u-a)^{s}}{s}, s>0$ in $(7)$ and $\phi(u)=-\frac{(b-u)^{s}}{s}, s>0$ in (8) then conformable $(k, s)$-fractional integrals.

8. For $\phi(u)=\frac{(u-a)^{s}}{s}, s>0$ in (7) and $\phi(u)=-\frac{(b-u)^{s}}{s}, s>0$ in (8), along with $k=1$, then conformable fractional integrals are achieved given in [14] are achieved.

Next, we give the definition of exponentially convex function.

Definition 5. [15,16] A function $\psi:[a, b] \rightarrow \mathbb{R}$ is said to be exponentially convex if for all $x, y \in[a, b]$ and $u \in[0,1]$, the following inequality holds;

$$
e^{\psi(u x+(1-u) y)} \leq u e^{\psi(x)}+(1-u) e^{\psi(y)} .
$$
follows;

The concept of exponentially $m$-convex functions was introduced by Rashid et al. in [17]. It is defined as

Definition 6. A function $\psi:[a, b] \rightarrow \mathbb{R}$ is said to be exponentially $m$-convex, where $m \in(0,1]$, if for all $x, y \in[a, b]$ and $u \in[0,1]$, the following inequality holds;

$$
e^{\psi(u x+m(1-u) y)} \leq u e^{\psi(x)}+m(1-u) e^{\psi(y)} .
$$

Remark 2. For $m=1$ in (10), (9) is achieved.

The aim of this research is to establish the bounds of the fractional integral operators defined in Definition 4. To establish these bounds exponentially $m$-convexity has been utilized. The established results provide all the possible outcomes of fractional integral operators given in remark (1). The breakup of this paper is: 
in Section 2, the first result provides the bounds of the generalized Riemann-Liouville $k$-fractional integral operators defined in Equations (7) and (8) for exponentially $m$-convex functions. The last result of Section 2 provides the fractional Hadamard type inequality. Furthermore, special cases of established results are also discussed. In Section 3, we give some applications of presented results.

\section{Main result}

First, we give the following bounds of the sum of the left-sided and right-sided fractional integral operators.

Theorem 1. Let $\psi, \phi:[a, b] \longrightarrow \mathbb{R}$ be two functions such that $\phi$ be differentiable and $\psi \in L[a, b]$ with $a<b$. Also let $\psi$ be exponentially m-convex, and $\phi$ be strictly increasing on $[a, b]$ with $\phi^{\prime} \in L_{1}[a, b]$. Then for $u \in[a, b]$ and $\sigma, \tau \geq k$, the following inequality holds;

$$
\begin{aligned}
& k\left(\Gamma_{k}(\sigma) \zeta_{\phi, a^{+}}^{\sigma, k} e^{\psi(u)}+\Gamma_{k}(\tau) \zeta_{\phi, b^{-}}^{\tau, k} e^{\psi(u)}\right) \leq \frac{(\phi(u)-\phi(a))^{\frac{\sigma}{k}-1}}{u-a}\left[(u-a)\left(m e^{\psi\left(\frac{u}{m}\right)} \phi(u)-e^{\psi(a)} \phi(a)\right)\right. \\
& \left.-\left(m e^{\psi\left(\frac{u}{m}\right)}-e^{\psi(a)}\right) \int_{a}^{u} \phi(\xi) d \xi\right]+\frac{(\phi(b)-\phi(u))^{\frac{\tau}{k}-1}}{b-u}\left[(b-u)\left(e^{\psi(b)} \phi(b)-m e^{\psi\left(\frac{u}{m}\right)} \phi(u)\right)\right. \\
& \left.-\left(e^{\psi(b)}-m e^{\psi\left(\frac{u}{m}\right)}\right) \int_{u}^{b} \phi(\xi) d \xi\right] .
\end{aligned}
$$

Proof. From exponentially $m$-convexity of $\psi$, we have

$$
e^{\psi(\xi)} \leq \frac{u-\xi}{u-a} e^{\psi(a)}+m \frac{\xi-a}{u-a} e^{\psi\left(\frac{u}{m}\right)} .
$$

Under given assumptions for the function $\phi$ : for $\sigma \geq k$, the following inequality holds;

$$
\phi^{\prime}(\xi)(\phi(u)-\phi(\xi))^{\frac{\sigma}{k}-1} \leq \phi^{\prime}(\xi)(\phi(u)-\phi(a))^{\frac{\sigma}{k}-1}, \quad \xi \in[a, u] \quad \text { and } u \in[a, b] .
$$

Multiplying (12) with (13) and integrating over $[a, u]$, we have

$$
\begin{aligned}
& \int_{a}^{u}(\phi(u)-\phi(\xi))^{\frac{\sigma}{k}-1} \phi^{\prime}(\xi) e^{\psi(\xi)} d \xi \\
& \leq \frac{(\phi(u)-\phi(a))^{\frac{\sigma}{k}-1}}{u-a}\left[e^{\psi(a)} \int_{a}^{u}(u-\xi) \phi^{\prime}(\xi) d \xi+m e^{\psi\left(\frac{u}{m}\right)} \int_{a}^{u}(\xi-a) \phi^{\prime}(\xi) d \xi\right] .
\end{aligned}
$$

By using (7), we get the following estimation;

$$
k \Gamma_{k}(\sigma) \zeta_{\phi, a^{+}}^{\sigma, k} e^{\psi(u)} \leq \frac{(\phi(u)-\phi(a))^{\frac{\sigma}{k}-1}}{u-a}\left[(u-a)\left(m e^{\psi\left(\frac{u}{m}\right)} \phi(u)-e^{\psi(a)} \phi(a)\right)-\left(m e^{\psi\left(\frac{u}{m}\right)}-e^{\psi(a)}\right) \int_{a}^{u} \phi(\xi) d \xi\right] .
$$

Again, from exponentially $m$-convexity of $\psi$ we have

$$
e^{\psi(\xi)} \leq \frac{\xi-u}{b-u} e^{\psi(b)}+m \frac{b-\xi}{b-u} e^{\psi\left(\frac{u}{m}\right)} .
$$

Now, for $\tau \geq k$, the following inequality holds;

$$
\phi^{\prime}(\xi)(\phi(\xi)-\phi(u))^{\frac{\tau}{k}-1} \leq \phi^{\prime}(\xi)(\phi(b)-\phi(u))^{\frac{\tau}{k}-1}, \quad \xi \in[u, b] \quad \text { and } \quad u \in[a, b] .
$$

Multiplying (15) with (16) and integrating over $[u, b]$, we have

$$
\int_{u}^{b}(\phi(\xi)-\phi(u))^{\frac{\tau}{k}-1} \phi^{\prime}(\xi) e^{\psi(\xi)} d \xi \leq \frac{(\phi(b)-\phi(u))^{\frac{\tau}{k}-1}}{b-u}\left[e^{\psi(b)} \int_{u}^{b}(\xi-u) \phi^{\prime}(\xi) d \xi+m e^{\psi\left(\frac{u}{m}\right)} \int_{u}^{b}(b-\xi) \phi^{\prime}(\xi) d \xi\right] .
$$


By using (8), we get the following estimation;

$k \Gamma_{k}(\tau) \zeta_{\phi, b^{-}}^{\tau, k} e^{\psi(u)} \leq \frac{(\phi(b)-\phi(u))^{\frac{\tau}{k}-1}}{b-u}\left[(b-u)\left(e^{\psi(b)} \phi(b)-m e^{\psi\left(\frac{u}{m}\right)} \phi(u)\right)-\left(e^{\psi(b)}-m e^{\psi\left(\frac{u}{m}\right)}\right) \int_{u}^{b} \phi(\xi) d \xi\right]$

From (14) and (17) we achieve (11).

Corollary 1. If we put $\sigma=\tau$ in (11), then following inequality holds;

$$
\begin{aligned}
& \zeta_{\phi, a^{+}}^{\sigma, k} e^{\psi(u)}+\zeta_{\phi, b^{-}}^{\sigma, k} e^{\psi(u)} \leq \frac{(\phi(u)-\phi(a))^{\frac{\sigma}{k}-1}}{k \Gamma_{k}(\sigma)(u-a)}\left[(u-a)\left(m e^{\psi\left(\frac{u}{m}\right)} \phi(u)-e^{\psi(a)} \phi(a)\right)-\left(m e^{\psi\left(\frac{u}{m}\right)}-e^{\psi(a)}\right) \int_{a}^{u} \phi(\xi) d \xi\right] \\
& +\frac{(\phi(b)-\phi(u))^{\frac{\sigma}{k}-1}}{k \Gamma_{k}(\sigma)(b-u)}\left[(b-u)\left(e^{\psi(b)} \phi(b)-m e^{\psi\left(\frac{u}{m}\right)} \phi(u)\right)-\left(e^{\psi(b)}-m e^{\psi\left(\frac{u}{m}\right)}\right) \int_{u}^{b} \phi(\xi) d \xi\right] .
\end{aligned}
$$

Corollary 2. Under the supposition of Theorem 1, let $m=1$. Then the following inequality for exponentially convex function holds:

$$
\begin{aligned}
& k\left(\Gamma_{k}(\sigma) \zeta_{\phi, a^{+}}^{\sigma, k} e^{\psi(u)}+\Gamma_{k}(\tau) \zeta_{\phi, b^{-}}^{\tau, k} e^{\psi(u)}\right) \\
& \leq \frac{(\phi(u)-\phi(a))^{\frac{\sigma}{k}-1}}{u-a}\left[(u-a)\left(e^{\psi(u)} \phi(u)-e^{\psi(a)} \phi(a)\right)-\left(e^{\psi(u)}-e^{\psi(a)}\right) \int_{a}^{u} \phi(\xi) d \xi\right] \\
& +\frac{(\phi(b)-\phi(u))^{\frac{\tau}{k}-1}}{b-u}\left[(b-u)\left(e^{\psi(b)} \phi(b)-e^{\psi(u)} \phi(u)\right)-\left(e^{\psi(b)}-e^{\psi(u)}\right) \int_{u}^{b} \phi(\xi) d \xi\right] .
\end{aligned}
$$

Corollary 3. Suppose $k=1$, then under under the supposition of Theorem 1, the following inequality for generalized Riemann-Liouville fractional integral operators holds;

$$
\begin{aligned}
& \Gamma(\sigma) \zeta_{\phi, a^{+}}^{\sigma} e^{\psi(u)}+\Gamma(\tau) \zeta_{\phi, b^{-}}^{\tau} e^{\psi(u)} \leq \frac{(\phi(u)-\phi(a))^{\sigma-1}}{u-a}\left[(u-a)\left(m e^{\psi\left(\frac{u}{m}\right)} \phi(u)-e^{\psi(a)} \phi(a)\right)\right. \\
& \left.-\left(m e^{\psi\left(\frac{u}{m}\right)}-e^{\psi(a)}\right) \int_{a}^{u} \phi(\xi) d \xi\right]+\frac{(\phi(b)-\phi(u))^{\tau-1}}{b-u}\left[(b-u)\left(e^{\psi(b)} \phi(b)-m e^{\psi\left(\frac{u}{m}\right)} \phi(u)\right)\right. \\
& \left.-\left(e^{\psi(b)}-m e^{\psi\left(\frac{u}{m}\right)}\right) \int_{u}^{b} \phi(\xi) d \xi\right] .
\end{aligned}
$$

Corollary 4. Suppose $\phi(u)=u$, then under the supposition of Theorem 1, the following inequality for Riemann-Liouville k-fractional integral operators holds;

$$
2 k\left(\Gamma_{k}(\sigma) \zeta_{a^{+}}^{\sigma, k} e^{\psi(u)}+\Gamma_{k}(\tau) \zeta_{b^{-}}^{\tau, k} e^{\psi(u)}\right) \leq(u-a)^{\frac{\sigma}{k}}\left[e^{\psi(a)}+m e^{\psi\left(\frac{u}{m}\right)}\right]+(b-u)^{\frac{\tau}{k}}\left[e^{\psi(b)}+m e^{\psi\left(\frac{u}{m}\right)}\right] .
$$

Corollary 5. Suppose $\phi(u)=u$ and $k=1$, then under the supposition of Theorem 1, the following inequality for Riemann-Liouville fractional integral operators holds;

$$
2\left(\Gamma(\sigma) \zeta_{a^{+}}^{\sigma} e^{\psi(u)}+\Gamma(\tau) \zeta_{b^{-}}^{\tau} e^{\psi(u)}\right) \leq(u-a)^{\sigma}\left[e^{\psi(a)}+m e^{\psi\left(\frac{u}{m}\right)}\right]+(b-u)^{\tau}\left[e^{\psi(b)}+m e^{\psi\left(\frac{u}{m}\right)}\right] .
$$

We need following lemma in the proof of next result.

Lemma 1. Let $\psi:[a, b] \rightarrow \mathbb{R}$ be an exponentially $m$-convex function. If $e^{\psi(\xi)}=e^{\psi\left(\frac{a+b-\tilde{\xi}}{m}\right)}$ then for $\xi \in[a, b]$ and $m \in(0,1]$, the following inequality holds;

$$
e^{\psi\left(\frac{a+b}{2}\right)} \leq \frac{1}{2}(m+1) e^{\psi(\xi)}
$$

Proof. We have

$$
\frac{a+b}{2}=\frac{1}{2}\left(\frac{\xi-a}{b-a} b+\frac{b-\xi}{b-a} a\right)+m \frac{1}{2}\left(\frac{\frac{\xi-a}{b-a} a+\frac{b-\xi}{b-a} b}{m}\right)
$$


Since $\psi$ is exponentially $m$-convex, we have

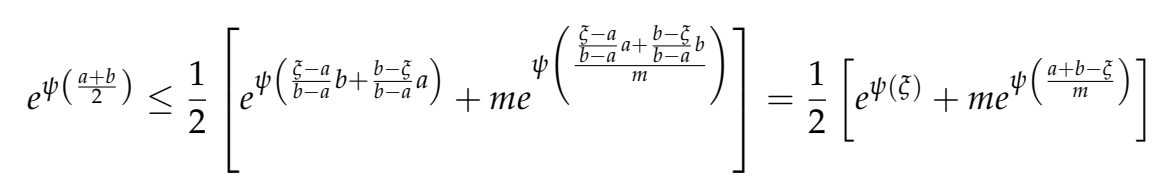

Using given condition $e^{\psi(\xi)}=e^{\psi\left(\frac{a+b-\tilde{\xi}}{m}\right)}$ in (23), the inequality (21) can be achieved.

Theorem 2. Let $\psi, \phi:[a, b] \subset[0, \infty) \longrightarrow \mathbb{R}$, be two functions such that $\phi$ be differentiable and $\psi \in L_{1}[a, b]$ with $a<b$. Also let $\psi$ be exponentially m-convex, $e^{\psi(\tilde{\xi})}=e^{\psi\left(\frac{a+b-\xi}{m}\right)}$ and $\phi$ be strictly increasing on $[a, b]$ with $\phi^{\prime} \in L_{1}[a, b]$. Then for $\xi \in[a, b]$ and $\sigma, v, k>0$, the following inequalities hold;

$$
\begin{aligned}
& \frac{2 k}{(m+1)} e^{\psi\left(\frac{a+b}{2}\right)}\left[\frac{(\phi(b)-\phi(a))^{\frac{\sigma}{k}+1}}{\sigma+k}+\frac{(\phi(b)-\phi(a))^{\frac{\tau}{k}+1}}{\tau+k}\right] \leq k\left(\Gamma_{k}(\sigma+k) \zeta_{\phi, b^{-}}^{\sigma+k, k} e^{\psi(a)}+\Gamma_{k}(\tau+k) \zeta_{\phi, a^{+}}^{\tau+k, k} e^{\psi(b)}\right) \\
& \leq \frac{(\phi(b)-\phi(a))^{\frac{\sigma}{k}}+(\phi(b)-\phi(a))^{\frac{\tau}{k}}}{(b-a)}\left[(b-a)\left(e^{\psi(b)} \phi(b)-m e^{\psi\left(\frac{a}{m}\right)} \phi(a)\right)-\left(e^{\psi(b)}-m e^{\phi\left(\frac{a}{m}\right)}\right) \int_{a}^{b} \phi(\xi) d \xi\right]
\end{aligned}
$$

Proof. Multiplying both sides of (21) with $(\phi(\xi)-\phi(a))^{\frac{\sigma}{k}} \phi^{\prime}(\xi)$ and integrating over $[a, b]$, we have

$$
e^{\psi\left(\frac{a+b}{2}\right)} \int_{a}^{b}(\phi(\xi)-\phi(a))^{\frac{\sigma}{k}} \phi^{\prime}(\xi) d \xi \leq \frac{1}{2}(m+1) \int_{a}^{b}(\phi(\xi)-\phi(a))^{\frac{\sigma}{k}} \phi^{\prime}(\xi) e^{\psi(\xi)} d \xi .
$$

By using (8), we get

$$
\frac{2 k(\phi(b)-\phi(a))^{\frac{\sigma}{k}+1}}{(\sigma+k)(m+1)} e^{\psi\left(\frac{a+b}{2}\right)} \leq k \Gamma_{k}(\sigma+k) \zeta_{\phi, b^{-}}^{\sigma+k, k} e^{\psi(a)} .
$$

Similarly, multiplying both sides of $(21)$ with $(\phi(b)-\phi(\xi))^{\frac{\tau}{k}} \phi^{\prime}(\xi)$ and integrating over $[a, b]$, one can get the following inequality:

$$
\frac{2 k(\phi(b)-\phi(a))^{\frac{\tau}{k}+1}}{(\tau+k)(m+1)} e^{\psi\left(\frac{a+b}{2}\right)} \leq k \Gamma_{k}(\tau+k) \zeta_{\phi, a^{+}}^{\tau+k, k} e^{\psi(b)}
$$

On the other hand from exponentially $m$-convexity of $\psi$, we have

$$
e^{\psi(\xi)} \leq \frac{\xi-a}{b-a} e^{\psi(b)}+m \frac{b-\xi}{b-a} e^{\psi\left(\frac{a}{m}\right)} .
$$

Under given assumptions for the function $\phi$ for $\sigma, k>0$, the following inequality holds;

$$
\phi^{\prime}(\xi)(\phi(\xi)-\phi(a))^{\frac{\sigma}{k}} \leq \phi^{\prime}(\xi)(\phi(b)-\phi(a))^{\frac{\sigma}{k}}, \quad \xi \in[a, b] .
$$

Multiplying (28) with (29) and integrating over $[a, b]$, we have

$$
\int_{a}^{b}(\phi(\xi)-\phi(a))^{\frac{\sigma}{k}} \phi^{\prime}(\xi) e^{\psi(\xi)} d \xi \leq \frac{(\phi(b)-\phi(a))^{\frac{\sigma}{k}}}{b-a}\left[e^{\psi(b)} \int_{a}^{b}(\xi-a) \phi^{\prime}(\xi) d \xi+m e^{\psi\left(\frac{a}{m}\right)} \int_{a}^{b}(b-\xi) \phi^{\prime}(\xi) d \xi\right] .
$$

By using (8), we get

$$
k \Gamma_{k}(\sigma+k) \zeta_{\phi, b^{-}}^{\sigma+k, k} e^{\psi(a)} \leq \frac{(\phi(b)-\phi(a))^{\frac{\sigma}{k}}}{b-a}\left[(b-a)\left(e^{\psi(b)} \phi(b)-m e^{\psi\left(\frac{a}{m}\right)} \phi(a)\right)-\left(e^{\psi(b)}-m e^{\phi\left(\frac{a}{m}\right)}\right) \int_{a}^{b} \phi(\xi) d \xi\right]
$$

Now for $\xi \in[a, b]$ and $\tau, k>0$, the following inequality holds true;

$$
\phi^{\prime}(\xi)(\phi(b)-\phi(\xi))^{\frac{\tau}{k}} \leq \phi^{\prime}(\xi)(\phi(b)-\phi(a))^{\frac{\tau}{k}} .
$$


Multiplying (28) with (31) and integrating over $[a, b]$, we have

$$
\int_{a}^{b}(\phi(b)-\phi(\xi))^{\frac{\tau}{k}} \phi^{\prime}(\xi) e^{\psi(\xi)} d \xi \leq \frac{(\phi(b)-\phi(a))^{\frac{\tau}{k}}}{b-a}\left[e^{\psi(b)} \int_{a}^{b}(\xi-a) \phi^{\prime}(\xi) d \xi+m e^{\psi\left(\frac{a}{m}\right)} \int_{a}^{b}(b-\xi) \phi^{\prime}(\xi) d \xi\right] .
$$

By using (8), we get

$$
k \Gamma_{k}(\tau+k) \zeta_{\phi, a^{+}}^{\tau+k, k} e^{\psi(b)} \leq \frac{(\phi(b)-\phi(a))^{\frac{\tau}{k}}}{b-a}\left[(b-a)\left(e^{\psi(b)} \phi(b)-m e^{\psi\left(\frac{a}{m}\right)} \phi(a)\right)-\left(e^{\psi(b)}-m e^{\phi\left(\frac{a}{m}\right)}\right) \int_{a}^{b} \phi(\xi) d \xi\right]
$$

By adding inequalities (30) and (32), second inequality (24) is achieved.

Corollary 6. If we put $\sigma=\tau$ in (24), we get following inequalities;

$$
\begin{aligned}
& \frac{4 k(\phi(b)-\phi(a))^{\frac{\sigma}{k}+1}}{(m+1)(\sigma+k)} e^{\psi\left(\frac{a+b}{2}\right)} \leq k \Gamma_{k}(\sigma+k)\left(\zeta_{\phi, b^{-}}^{\sigma+k, k} e^{\psi(a)}+\zeta_{\phi, a^{+}}^{\sigma+k, k} e^{\psi(b)}\right) \\
& \leq \frac{2(\phi(b)-\phi(a))^{\frac{\sigma}{k}}}{(b-a)}\left[(b-a)\left(e^{\psi(b)} \phi(b)-m e^{\psi\left(\frac{a}{m}\right)} \phi(a)\right)-\left(e^{\psi(b)}-m e^{\phi\left(\frac{a}{m}\right)}\right) \int_{a}^{b} \phi(\xi) d \xi\right] .
\end{aligned}
$$

Corollary 7. Suppose $m=1$, then under the supposition of Theorem 2, the following inequalities for exponentially convex function hold;

$$
\begin{aligned}
& k e^{\psi\left(\frac{a+b}{2}\right)}\left[\frac{(\phi(b)-\phi(a))^{\frac{\sigma}{k}+1}}{\sigma+k}+\frac{(\phi(b)-\phi(a))^{\frac{\tau}{k}+1}}{\tau+k}\right] \leq k\left(\Gamma_{k}(\sigma+k) \zeta_{\phi, b^{-}}^{\sigma+k, k} e^{\psi(a)}+\Gamma_{k}(\tau+k) \zeta_{\phi, a^{+}}^{\tau+k, k} e^{\psi(b)}\right) \\
& \leq \frac{(\phi(b)-\phi(a))^{\frac{\sigma}{k}}+(\phi(b)-\phi(a))^{\frac{\tau}{k}}}{(b-a)}\left[(b-a)\left(e^{\psi(b)} \phi(b)-e^{\psi(a)} \phi(a)\right)-\left(e^{\psi(b)}-e^{\phi(a)}\right) \int_{a}^{b} \phi(\xi) d \xi\right] .
\end{aligned}
$$

Corollary 8. Suppose $k=1$, then under the supposition of Theorem 2, the following inequalities for generalized Riemann-Liouville fractional integral operators hold;

$$
\begin{aligned}
& \frac{2}{(m+1)} e^{\psi\left(\frac{a+b}{2}\right)}\left[\frac{(\phi(b)-\phi(a))^{\sigma+1}}{\sigma+1}+\frac{(\phi(b)-\phi(a))^{\tau+1}}{\tau+1}\right] \leq \Gamma(\sigma+1) \zeta_{\phi, b^{-}}^{\sigma+1} e^{\psi(a)}+\Gamma(\tau+1) \zeta_{\phi, a^{+}}^{\tau+1} e^{\psi(b)} \\
& \leq \frac{(\phi(b)-\phi(a))^{\sigma}+(\phi(b)-\phi(a))^{\tau}}{(b-a)}\left[(b-a)\left(e^{\psi(b)} \phi(b)-m e^{\psi\left(\frac{a}{m}\right)} \phi(a)\right)-\left(e^{\psi(b)}-m e^{\phi\left(\frac{a}{m}\right)}\right) \int_{a}^{b} \phi(\xi) d \xi\right] .
\end{aligned}
$$

Corollary 9. Suppose $\phi(u)=u$, then under the supposition of Theorem 2, the following inequalities for Riemann-Liouville $k$-fractional integral operators hold;

$$
\begin{aligned}
& \frac{2 k}{(m+1)} e^{\psi\left(\frac{a+b}{2}\right)}\left[\frac{(b-a)^{\frac{\sigma}{k}+1}}{\sigma+k}+\frac{(b-a)^{\frac{\tau}{k}+1}}{\tau+k}\right] \leq k\left(\Gamma_{k}(\sigma+k) \zeta_{b^{-}}^{\sigma+k, k} e^{\psi(a)}+\Gamma_{k}(\tau+k) \zeta_{a^{+}}^{\tau+k, k} e^{\psi(b)}\right) \\
& \leq \frac{(b-a)^{\frac{\sigma}{k}+1}+(b-a)^{\frac{\tau}{k}+1}}{2}\left[e^{\psi(b)}+m e^{\psi\left(\frac{a}{m}\right)}\right]
\end{aligned}
$$

Corollary 10. Suppose $\phi(u)=u$ and $k=1$, then under the supposition of Theorem 2, the following inequalities for Riemann-Liouville fractional integral operators hold;

$$
\begin{aligned}
& \frac{2}{(m+1)} e^{\psi\left(\frac{a+b}{2}\right)}\left[\frac{(b-a)^{\sigma+1}}{\sigma+1}+\frac{(b-a)^{\tau+1}}{\tau+1}\right] \leq \Gamma(\sigma+1) \zeta_{b^{-}}^{\sigma+1} e^{\psi(a)}+\Gamma(\tau+1) \zeta_{a^{+}}^{\tau+1} e^{\psi(b)} \\
& \leq \frac{(b-a)^{\sigma+1}+(b-a)^{\tau+1}}{2}\left[e^{\psi(b)}+m e^{\psi\left(\frac{a}{m}\right)}\right]
\end{aligned}
$$

\section{Applications}

In this section, we give the applications of the results proved in previous section. 
Theorem 3. Under the assumptions of Theorem 1, we have

$$
\begin{aligned}
& k\left(\Gamma_{k}(\sigma) \zeta_{\phi, a^{+}}^{\sigma, k} e^{\psi(b)}+\Gamma_{k}(\tau) \zeta_{\phi, b^{-}}^{\tau, k} e^{\psi(a)}\right) \leq \frac{(\phi(b)-\phi(a))^{\frac{\sigma}{k}-1}}{b-a}\left[(b-a)\left(m e^{\psi\left(\frac{b}{m}\right)} \phi(b)-e^{\psi(a)} \phi(a)\right)\right. \\
& \left.-\left(m e^{\psi\left(\frac{b}{m}\right)}-e^{\psi(a)}\right) \int_{a}^{b} \phi(\xi) d \xi\right]+\frac{(\phi(b)-\phi(a))^{\frac{\tau}{k}-1}}{b-a}\left[(b-a)\left(e^{\psi(b)} \phi(b)-m e^{\psi\left(\frac{a}{m}\right)} \phi(a)\right)\right. \\
& \left.-\left(e^{\psi(b)}-m e^{\psi\left(\frac{a}{m}\right)}\right) \int_{a}^{b} \phi(\xi) d \xi\right] .
\end{aligned}
$$

Proof. If we put $u=a$ in (11), we get

$$
k \Gamma_{k}(\tau) \zeta_{\phi, b^{-}}^{\tau, k} e^{\psi(a)} \leq \frac{(\phi(b)-\phi(a))^{\frac{\tau}{k}-1}}{b-a}\left[(b-a)\left(e^{\psi(b)} \phi(b)-m e^{\psi\left(\frac{a}{m}\right)} \phi(a)\right)-\left(e^{\psi(b)}-m e^{\psi\left(\frac{a}{m}\right)}\right) \int_{a}^{b} \phi(\xi) d \xi\right] .
$$

If we put $u=b$ in (11), we get

$$
k \Gamma_{k}(\sigma) \zeta_{\phi, a^{+}}^{\sigma, k} e^{\psi(b)} \leq \frac{(\phi(b)-\phi(a))^{\frac{\sigma}{k}-1}}{b-a}\left[(b-a)\left(m e^{\psi\left(\frac{b}{m}\right)} \phi(b)-e^{\psi(a)} \phi(a)\right)-\left(m e^{\psi\left(\frac{b}{m}\right)}-e^{\psi(a)}\right) \int_{a}^{b} \phi(\xi) d \xi\right] .
$$

By adding inequalities (37) and (38), inequality (36) can be achieved.

Corollary 11. If we put $\sigma=\tau$ in (36), then the following inequality holds;

$$
\begin{aligned}
& \zeta_{\phi, a^{+}}^{\sigma, k} e^{\psi(b)}+\zeta_{\phi, b^{-}}^{\sigma, k} e^{\psi(a)} \leq \frac{(\phi(b)-\phi(a))^{\frac{\sigma}{k}-1}}{k \Gamma_{k}(\sigma)(b-a)}\left[( b - a ) \left(\left(m e^{\psi\left(\frac{b}{m}\right)} \phi(b)-e^{\psi(a)} \phi(a)\right)\right.\right. \\
& \left.\left.+\left(e^{\psi(b)} \phi(b)-m e^{\psi\left(\frac{a}{m}\right)} \phi(a)\right)\right)-\left(\left(m e^{\psi\left(\frac{b}{m}\right)}-e^{\psi(a)}\right)+\left(e^{\psi(b)}-m e^{\psi\left(\frac{a}{m}\right)}\right)\right) \int_{a}^{b} \phi(\xi) d \xi\right]
\end{aligned}
$$

Corollary 12. If we put $\sigma=k=m=1$ and $\phi(u)=u$ in (39), then the following inequality holds;

$$
\frac{1}{b-a} \int_{a}^{b} e^{\psi(\xi)} d \xi \leq \frac{e^{f(a)}+e^{f(b)}}{2} .
$$

Remark 3. Similar relation can be achieved by applying Theorem 2, so we leave it for readers.

Author Contributions: Both authors contributed equally to the writing of this paper. All authors read and approved the final manuscript.

Conflicts of Interest: "The authors declare no conflict of interest."

\section{References}

[1] Farid, G. (2019). Some Riemann-Liouville fractional integral inequalities for convex functions. The Journal of Analysis, 27(4), 1095-1102.

[2] Chen, H., \& Katugampola, U. N. (2017). Hermite-Hadamard and Hermite-Hadamard-Fejér type inequalities for generalized fractional integrals. Journal of Mathematical Analysis and Applications, 446(2), 1274-1291.

[3] Farid, G., \& Abbas, G. (2018). Generalizations of some fractional integral inequalities for m-convex functions via generalized Mittag-Leffler function. Studia Universitatis Babes-Bolyai Mathematica, 63(1), 23-35.

[4] Farid, G., Khan, K. A., Latif, N., Rehman, A. U., \& Mehmood, S. (2018). General fractional integral inequalities for convex and m-convex functions via an extended generalized Mittag-Leffler function. Journal of inequalities and applications, 2018(1), 1-12.

[5] Farid, G., Nazeer, W., Saleem, M. S., Mehmood, S., \& Kang, S. M. (2018). Bounds of Riemann-Liouville fractional integrals in general form via convex functions and their applications. Mathematics, 6(11), 248.

[6] Kang, S. M., Farid, G., Nazeer, W. \& Mehmood, S. (2019). (h, m)-convex functions and associated fractional Hadamard and Fejér-Hadamard inequalities via an extended generalized Mittag-Leffler function. Journal of Inequalities and Applications, 2019, 78 pp.

[7] Kwun, Y. C., Farid, G., Nazeer, W., Ullah, S., \& Kang, S. M. (2018). Generalized Riemann-Liouville k-Fractional Integrals Associated With Ostrowski Type Inequalities and Error Bounds of Hadamard Inequalities. IEEE access, 6, 64946-64953. 
[8] Mehmood, S., \& Farid, G. (2019). $m$-Convex functions associated with bounds of $k$-fractional integrals. Advances in Inequalities and Applications, 2020, Article-ID 1.

[9] Mubeen, S., \& Habibullah, G. M. (2012). k-Fractional integrals and application. International Journal of Contemporary Mathematical Sciences, 7(2), 89-94.

[10] Ullah, S., Farid, G., Khan, K. A., Waheed, A., \& Mehmood, S. (2019). Generalized fractional inequalities for quasi-convex functions. Advances in Difference Equations, 2019(1), 15.

[11] Kilbas, A. A. A., Srivastava, H. M., \& Trujillo, J. J. (2006). Theory and applications of fractional differential equations (Vol. 204). Elsevier Science Limited.

[12] Sarikaya, M. Z., Dahmani, Z., KIRIS, M. E., \& Ahmad, F. (2016). (k,s)-Riemann-Liouville fractional integral and applications. Hacettepe Journal of Mathematics and Statistics, 45(1), 77-89.

[13] Khan, T. U., \& Khan, M. A. (2019). Generalized conformable fractional operators. Journal of Computational and Applied Mathematics, 346, 378-389.

[14] Jarad, F., Ugurlu, E., Abdeljawad, T., \& Baleanu, D. (2017). On a new class of fractional operators. Advances in Difference Equations, 2017(1), 247.

[15] Antczak, T. (2001). ( $p, r)$-invex sets and functions. Journal of Mathematical Analysis and Applications, 263(2), 355-379.

[16] Dragomir, S. S., \& Gomm, I. (2015). Some Hermite-Hadamard type inequalities for functions whose exponentials are convex. Studia Universitatis Babes-Bolyai Mathematica, 60(4), 527-534.

[17] Rashid, S., Noor, M. A., \& Noor, K. I. (2019). Fractional exponentially $m$-convex functions and inequalities. International Journal of Analysis and Applications, 17(3), 464-478.

(c) 2020 by the authors; licensee PSRP, Lahore, Pakistan. This article is an open access article distributed under the terms and conditions of the Creative Commons Attribution (CC-BY) license (http://creativecommons.org/licenses/by/4.0/). 